\title{
Per inserire neologismi formati da nomi propri nei vocabolari c'è tempo
}

\section{Paolo D'Achille}

PUBBLICATO: 28 GIUGNO 2019

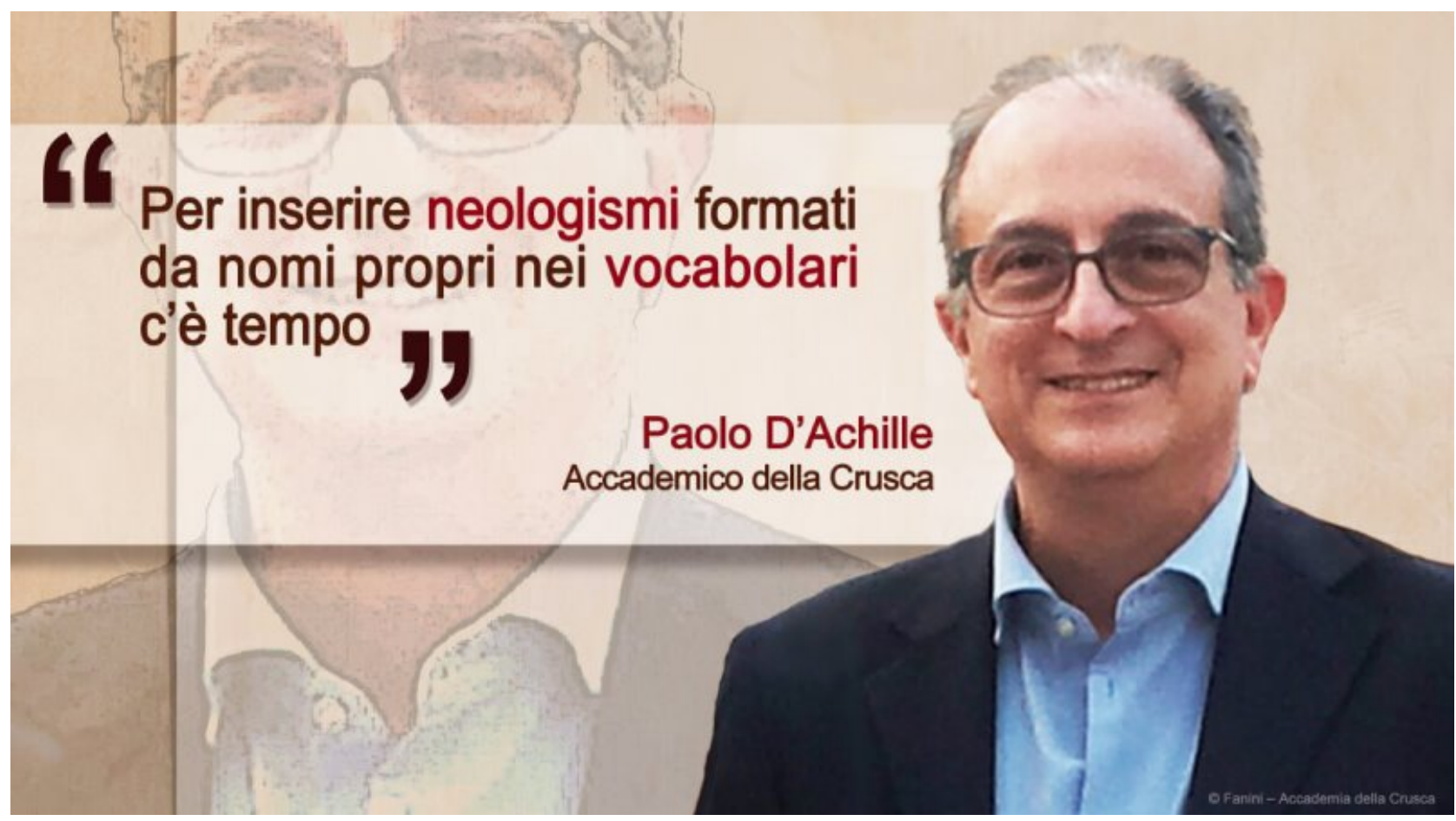

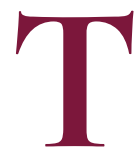
ra le differenze che vengono solitamente indicate tra il dizionario e l'enciclopedia, oltre al fatto che il primo tende a spiegare le parole e la seconda punta a descrivere le cose che le parole indicano, c'è anche una particolarità relativa all'estensione del lemmario: quello del dizionario esclude infatti categoricamente i nomi propri, che invece sono compresi nelle enciclopedie, tra le cui entrate figurano moltissimi personaggi storici, poeti, filosofi, scienziati, nonché città, monti, frumi, continenti, paesi, monumenti, ecc. Il dizionario include inoltre parole che non si trovano normalmente tra le voci enciclopediche: quelle appartenenti ad alcune specifiche categorie grammaticali (articoli, pronomi, avverbi, congiunzioni, preposizioni, ma anche verbi e aggettivi) e molti derivati da una stessa base. È vero che i confini tra i due tipi testuali si sono progressivamente ridotti e che non mancano esempi di commistioni (pensiamo a opere come il Dizionario enciclopedico italiano della Treccani), ma, in linea di massima, questa distinzione si può considerare ancora valida, specie per quanto riguarda i nomi propri, che continuano a rimanere esclusi dai dizionari.

In realtà, la tradizionale distinzione tra i nomi comuni (che indicano insiemi di elementi omogenei) e i nomi propri (che hanno invece una funzione individuante, perché riferiti a un unico essere o a una sola cosa) è stata talvolta messa in discussione anche sul piano teorico. Inoltre, dal punto di vista storico, le due categorie non costituiscono due insiemi non comunicanti: da un lato ci sono stati nomi comuni che, nel corso tempo, sono diventati nomi propri (è il caso di molti toponimi, che originariamente erano motivati, e anche di vari nomi di mestiere, divenuti cognomi), dall'altro si è avuto quel processo inverso (studiato magistralmente da Bruno Migliorini) per cui alcuni nomi propri sono diventati nomi comuni, per lo più in seguito a fenomeni di antonomasia (particolarmente frequenti con i nomi di 
persona: per es. adone, mecenate, dongiovanni) o di ellissi (soprattutto per i nomi di alimenti e di bevande: formaggi come asiago, gorgonzola, spumanti come asti, prosecco ecc.).

Un altro motivo di avvicinamento tra le due categorie è costituito dal fatto che i nomi propri sono spesso alla base di nomi comuni: si pensi anzitutto ai derivati, tra cui spiccano per numerosità gli etnici, che indicano gli abitanti di una città o di un determinato territorio, ma anche ad alcuni composti (come bagnomaria) e in particolare alle cosiddette polirematiche (gruppi di parole che hanno un significato unitario), tra le quali non mancano espressioni che contengono al loro interno un nome proprio. Un dizionario come il GRADIT, grazie anche all'ampiezza del lemmario, è stato molto accogliente nei confronti dei nomi propri, includendo nel lemmario moltissimi etnici (quelli di quasi tutti i centri abitati italiani e anche di stati e città esteri) e ammettendovi anche un cerrto numero di antroponimi e toponimi (Cartesio, san Pietro, Roma) per registrare (anche) sotto queste entrate le polirematiche in cui sono i nomi propri inclusi, come diavoletto di Cartesio, pesce san Pietro, marcia su Roma. Gli altri dizionari ospitano solo alcuni etnici (selezionando almeno quelli dei centri maggiori o di uso più frequente) e non i nomi propri, segnalando alcune polirematiche che li comprendono sotto la voce del nome comune che ne costituisce la testa (cosi, per es., diavoletto di Cartesio nello Zingarelli 2019 figura s.v. diavoletto).

Con specifico riferimento ai derivati da nomi di persone, va detto che la loro presenza nell'italiano contemporaneo è molto alta, in particolare nella cronaca politica e nel mondo dello spettacolo. Lo documentano i repertori di neologismi, che del resto si basano prevalentemente sui giornali, ma ognuno di noi nel proprio uso personale crea, occasionalmente, formazioni del genere, per riferirsi a parenti, amici e conoscenti. Moltissimi sono i prefissi, gli affissi e i confissi che possono formare nomi comuni da nomi propri e tra le nuove formazioni abbiamo aggettivi, nomi e anche verbi. Tra i prefissi che si legano a nomi propri si ricordino almeno pre-, post-, anti- e pro-. Ancora più numerosi sono i suffissati, tra i quali (senza pretendere di fornirne una lista completa) si possono citare i numerosi derivati aggettivali (alcuni dei quali frequentemente convertiti in nomi) in -esco (baudesco), -iano (dalemiano), -ino (travoltino) e -ista (dipietrista); i derivati in -ista si legano spesso a formazioni nominali in -ismoche indicano movimenti, credenze e tendenze, ma anche correnti di partito, ecc. (beppegrillismo). Non mancano derivati da nomi propri tra i verbi formati con i suffissi -eggiare (che si usa spessissimo per indicare che qualcuno sembra imitare nel comportamento un'altra persona: Salvini renzeggia 'si comporta come Renzi') e -izzare (negli anni Novanta la RAI si è berlusconizzata 'ha trasmesso programmi simili a quelli che si vedevano sulle reti di Berlusconi') e tra i corrispondenti nomi d'azione in -mento e -zione. Numerosissime sono anche le formazioni in -ata che indicano, quasi sempre con una connotazione negativa, un atto considerato tipico della persona il cui nome è alla base del derivato (si pensi alle cassanate, dal calciatore Antonio Cassano, di cui si parlava qualche anno fa).

I derivati da nomi propri trovano spazio, come si è detto, nei dizionari di neologismi, ma solo di rado riescono a entrare stabilmente nella lingua e/o nella lessicografia, in rapporto allimportanza del personaggio a cui si riferiscono (importanza che in genere si può appurare solo a distanza di tempo). La lessicografia storica accoglieva questi lessemi cum grano salis (considerandoli, al pari degli etnici, nomi propri a tutti gli effetti e quindi escludendoli di norma dai lemmari). La lessicografia moderna è piu aperta e tende a registrare soprattutto le parole che si sono allontanate sul piano semantico dal nome proprio da cui derivano per indicare qualcosa di più generale: è il caso per esempio di kafkiano e felliniano, che non si riferiscono solo al grande scrittore praghese di lingua tedesca e al noto regista italiano, ma anche a situazioni o atmosfere angosciose e allucinanti in un caso, a toni surreali e onirici nell'altro (che richiamano, rispettivamente, gli scritti del primo e i film del secondo).

E stato il successo di una neoformazione recente tratta da un nome proprio che ci ha spinto a dedicare questo tema del mese ai nomi propri e ai loro derivati (già oggetto del fascicolo 56 della "Crusca per 
voi"). All'Accademia sono infatti arrivate di recente, a ondate, segnalazioni del neologismo vascologia, con la richiesta di valutare la parola in vista del suo possibile inserimento nei dizionari. Il termine, che indica lo studio della vita e delle opere di Vasco Rossi, sembra sia stato ideato da Vittoria Chiarenza, autrice di vari testi dedicati al rocker emiliano, che per varie generazioni di fans è anche maestro di un particolare stile di vita e di comportamento. È appena il caso di notare come la base sia in questo caso (ma non si tratta dell'unico esempio del genere) costituita dal (pre)nome dell'artista e non dal cognome, il che si spiega, tra le altre cose, col fatto che Rossi è il cognome più frequente in italiano, e come tale poco caratterizzante.

Anzitutto, è questa l'occasione per ribadire ai tanti che ci seguono e che ci interpellano che la scelta di "mettere una parola" nel vocabolario non spetta all'Accademia (che al momento non sta predisponendo un nuovo vocabolario dell'italiano di oggi): sono le redazioni delle varie case editrici che rinnovano periodicamente (a volte con scadenze persino annuali) le loro opere lessicografiche a rivedere il lemmario, a raccogliere parole nuove e a decidere quali di esse meritino di essere incluse nell'edizione più aggiornata e quali no perché destinate probabilmente a un rapido declino. L'Accademia della Crusca, all'interno del Servizio di Consulenza sulla lingua contemporanea, si occupa sì di neologismi, anche sulla base delle segnalazioni ricevute, ma col solo scopo di chiarirne l'origine, spiegarne il significato e precisarne gli àmbiti d'uso (spesso si tratta di anglismi).

Tornando alla vascologia, è vero che perfino i confissi (o suffissoidi) -logia e -logo, che indicano rispettivamente lo studio sistematico (spesso teorico) di un ben preciso campo concettuale e l'esperto di una particolare disciplina, possono essere aggiunti a nomi propri di persone ritenute meritevoli di trattazioni specifiche, ma in genere si tratta di formazioni occasionali, spesso scherzose o comunque di uso circoscritto, non destinate a entrare stabilmente nel lessico generale. Infatti, tra le oltre 800 formazioni in -logia comprese nel GRADIT ce ne sono alcune (poche) che hanno per base un toponimo o un etnico (albanologia 'studio della lingua e della letteratura albanese', assiriologia, cremlinologia 'studio e interpretazione della politica dell'Unione Sovietica e, dopo il I99I, della Russia', egittologia, etruscologia, sinologia 'studio di lingua, letteratura, cultura cinese'), ma quelle che partono da un nome proprio sono davvero eccezionali. A parte due termini della teologia cattolica, cristologia e mariologia, le uniche altre due che sono riuscito a individuare sono dantologia e petrarcologia, che indicano rispettivamente il complesso degli studi su Dante e di quelli su Petrarca, studi, gli uni e gli altri, numerosissimi e distribuiti nel corso di vari secoli. Visto allora che neppure le Tre Corone sono presenti al completo, perché manca il derivato da Boccaccio, direi che per l'inserimento nei dizionari italiani di altre formazioni con -logia che hanno per base il nome di un personaggio vivente c'e ancora tempo.

\section{Cita come:}

Paolo D'Achille, Per inserire neologismi formati da nomi propri nei vocabolari c'è tempo , "Italiano digitale", 2019, IX, 2019/2 (aprile-giugno)

DOI: $10.35948 / 2532-9006 / 2020.3152$

Copyright 2019 Accademia della Crusca

Pubblicato con licenza creative commons CC BY-NC-ND 